\title{
The relationship of human acellular dermal matrix thickness on complication rate and patient-reported outcomes in implant- based immediate breast reconstruction
}

\author{
Seung Eun Hong, Jung-Hoon Kim \\ Department of Plastic and Reconstructive Surgery, Ewha Womans University Mokdong Hospital, College of Medicine, Ewha Womans University, \\ Seoul, Korea \\ Contributions: (I) Conception and design: SE Hong; (II) Administrative support: SE Hong; (III) Provision of study materials or patients: SE Hong; \\ (IV) Collection and assembly of data: All authors; (V) Data analysis and interpretation: All authors; (VI) Manuscript writing: All authors; (VII) Final \\ approval of manuscript: All authors. \\ Correspondence to: Seung Eun Hong, MD, PhD, JD. Department of Plastic and Reconstructive Surgery, Ewha Womans University Mokdong Hospital, \\ College of Medicine, Ewha Womans University, 1071, Anyangcheon-ro, Yangcheon-Gu, Seoul 07985, Korea. Email: monkeyhong@hanmail.net.
}

\begin{abstract}
Background As it can be said that prosthetic breast reconstruction has been increased by the use of acellular dermal matrices (ADMs), ADMs are widely used in implant reconstruction. A large variety types of $\mathrm{ADMs}$ are now produced, but there is a paucity of data comparing the ADM products directly. We evaluated the effects of ADM thickness on complication rates and patient satisfaction after dual-plane subpectoral ADM-assisted implant breast reconstruction.

Methods: A retrospective study of patients who underwent immediate implant breast reconstruction using ADM was performed. We compared clinical course and postoperative outcomes for two ADM groups with different thicknesses [thin ADMs, 0.6-1.5 mm (group 1) vs. thick ADMs, 1.5-3.0 mm (group 2)] based on the incidence of complications and the duration of drainage. Patient satisfaction was also evaluated using the Breast Questionnaire (BREAST-Q), 6 months after surgery.

Results: A total of 51 patients were included in the study (group 1, n=21; group 2, n=30). Patient demographics were similar between the two groups, and no difference in postoperative complication rate $(\mathrm{P}>0.05)$ and Jackson-Pratt (JP) drainage durations $(\mathrm{P}>0.05)$. On regression analysis, ADM thickness was not an independent factor for any complication subtype. There were also no significant differences in BREAST-Q results, across all metrics, between the study groups.

Conclusions: In dual plane subpectoral implant placement breast reconstruction with an inferior ADMsling, the thickness of ADM was not a factor in determining postoperative complications and patient satisfaction. Therefore, it is possible to select the thickness according to the surgeon's preference.
\end{abstract}

Keywords: Acellular dermal matrix (ADM); breast reconstruction; silicone implant; thickness

Submitted May 29, 2020. Accepted for publication Nov 17, 2020.

doi: $10.21037 /$ gs-20-534

View this article at: http://dx.doi.org/10.21037/gs-20-534

\section{Introduction}

Breast cancer is one of the major malignancies in women (1). However, breast cancer is known to have high cure rate and low mortality rate through early diagnosis and active treatment with advanced treatment modalities (2). This increased survival has led to changes in surgical approaches and enhanced efforts to improve the quality of life after surgery. Since skin (or nipple)-sparing mastectomies have proven to be oncologically safe, more patients with invasive breast cancer now undergo breast reconstruction $(3,4)$. 
Social consensus on the need for breast reconstruction has also been drawn up, and thus, breast reconstruction surgery can benefit from health insurance in South Korea.

Reconstruction using implants (or tissue expanders), which comprise the majority of breast reconstructions, is a surgical technique that can give great esthetic satisfaction to patients in a simple way that does not require special surgical technique. Inserting the implant or tissue expander under the pectoralis muscle provides an excellent cosmetic result, whereas total submuscular reconstruction was associated with postoperative pain, the potential for breast animation deformity, deviation of the breast shape with unclear inframammary fold, and lower pole fullness insufficiency $(5,6)$. An alternative option is incomplete muscular cover (dual plane), where the lower pole is covered only by the skin flap (7). This allows for the expansion of the inferior pole and creation of a natural, ptotic breast shape. However, the inferior pole of the implant can be left without muscle coverage, leaving a significant exposure risk; this often leads to inferior migration of the implant and obvious rippling. Therefore, several techniques are described to improve implant coverage in the lower pole. More recently, in the breast reconstruction method that involves using an implant, acellular dermal matrix (ADM) is being inserted between the implant and the breast skin flap. ADM is a skin substitute which is proposed to (I) improve control over the placement of the inframammary fold, (II) enhance the shape of the reconstructed breast, (III) allow for proper use of the available mastectomy skin, and (IV) reduce the need for explantation and the incidence of capsular contracture.

$\mathrm{ADM}$ is a collagen substrate generated by decellularization while preserving the intact extracellular skin matrix that is used for soft tissue coverage. It can be prepared from any organ or tissue, including human, bovine, and porcine tissues, depending on the purpose of clinical application. During breast reconstruction, acellular human dermis products are primarily used in the setting of implant reconstruction. ADMs which are derived from cadaveric tissues are decellularized and often terminally sterilized, but proprietary decellularization and sterilization techniques vary among manufacturing companies (8). For example, in recent years, a cross-linking processing technique using electron beams has been applied to ADM. Cross-linked ADMs have different physical properties from non-cross-linked human dermis allografts; therefore, it may affect the surgical outcome. In addition, in South Korea, ADM can be produced as a customized product, and thus, the thickness and the size of ADM is determined according to the request of the surgeon. Surgeons have different personal preferences for the type of ADM they want to use, and there is still a lack of research about how the surgical outcome is affected due to these features. This work aimed to provide more reliable evidence that the thickness of ADM affects the surgical outcome and patient satisfaction as well as provide a specific reference value for reconstructive surgeons. In addition, we intended to analyze the factors which may be associated with complications.

We present the following article in accordance with the STROBE reporting checklist (available at http://dx. doi. org/10. 21037/gs-20-534).

\section{Methods}

\section{Study design}

We performed a retrospective review of medical records of patients who underwent direct-to-implant (DTI) breast reconstruction using ADM. This study was approved by the Institutional Review Board of Ewha Mokdong Hospital (IRB No. 2020-05-043) and was performed in accordance with the principles of the Declaration of Helsinki (as revised in 2013). The requirement of obtaining individual consent was waived for this retrospective analysis. Four-year (January 2015 to December 2018) data on all DTI breast reconstructions, including details on thick and thin ADMs, were included. We defined "thick" $\mathrm{ADM}$ as $\geq 1.5 \mathrm{~mm}$ and "thin" ADM as $<1.5 \mathrm{~mm}$ (the thickness was based on what is recorded in the product, not measured separately). In other words, it was divided into a thin ADM group and a thick ADM group based on $1.5 \mathrm{~mm}$. Individual charts of all patients were retrospectively reviewed to extract data on demographic information, surgery-related characteristics, ADM thickness, complications [hematoma, seroma, cellulitis, flap necrosis, infections (requiring intravenous antibiotics), and revision required], and total number of days before Jackson-Pratt (JP) drain removal.

\section{Surgical technique}

When mastectomy was performed, by detaching the inferior costal origin of the pectoralis major muscle, we created subpectoral dual plane pockets. The wounds were irrigated with gentamicin and cetrazole, and the chosen ADM was placed to cover the inferior pole of the implant. In all cases, we used MegaDerm ${ }^{\circledast}$ (L\&C Bio, Seoul, Republic of Korea) 
as the $\mathrm{ADM}$ material for breast reconstruction, regardless of the ADM thickness required. MegaDerm is an ADM allograft classified as banked human tissue. After positioning the ADM between the inferior border of the pectoralis major muscle and the inframammary fold, VICRYL ${ }^{\circledR}$ suture (Ethicon Inc., Somerville, NJ, USA) fixation was done. Anatomical implants (Mentor Worldwide LLC., Santa Barbara, CA, USA; Mentor MemoryGel ${ }^{\circledR}$ ) were placed in the subpectoral-ADM pocket. Two drains were placed, and skin flaps were closed following the standard method. The drains were removed when the amount of drainage was less than $20 \mathrm{~mL} /$ day for 2 consecutive days. Each reconstructive surgery was performed by a single experienced plastic surgeon for ensuring consistency in technique of handling $\mathrm{ADM}$, drain placement, and postoperative drain management and removal.

\section{Patient satisfaction}

Patient-reported outcomes and the effect of breast surgery on the patient's quality of life were evaluated using Breast Questionnaire (BREAST-Q) (9). The questionnaire was administered to patients who visited the hospital 6 months after the surgery; a telephone survey was conducted if a visit was difficult.

\section{Statistical analysis}

R language version 3.3.3 (R Foundation for Statistical Computing, Vienna, Austria), T\&F program version 2.2 (YooJin BioSoft, Seoul, Republic of Korea), and IBM Statistical Package for the Social Sciences version 22 (IBM Corp., New York, USA) were used for all statistical analyses. When continuous variables were normally distributed, the mean difference test between ADM type (thick $v s$. thin) was performed using the Student's $t$-test or Welch's $t$-test. For non-normally distributed variables, the Mann-Whitney $U$ test was used. For categorical variables, the Chi-squared test or Fisher's exact test was performed to test the hypothesis of association between ADM type (thick vs. thin) and other variables, as appropriate using contingency tables. Linear regression analysis was performed to analyze the effect of each clinical measurement on the time to JP drain removal. For analyzing the combined effect of more than two variables on the response of ADM thickness, multivariable linear regression analysis was performed. Binary logistic regression analysis was performed to analyze the effect of each clinical measurement on the binary response of complications. For analyzing the combined effect of more than two variables on binary response, multivariable logistic regression analysis was performed.

\section{Results}

A total of 51 DTI breast reconstructions were performed. Reconstruction with thin $\mathrm{ADM}$ was performed in 21 cases $(41.2 \%)$ and with thick ADM in 30 cases $(58.8 \%$; Table 1$)$. The mean age of patients was $48.9 \pm 10.0$ years. The mean volume of implants inserted was $263.2 \pm 100.3 \mathrm{~cm}^{3}$, and the mean follow-up period was 13.5 months (range, 642 months). Patient demographics were similar between the thin and thick ADM groups and showed no statistically significant differences. There were no differences in the rates of comorbidities, including diabetes, or hypertension. Demographic data for the subjects are listed in Table 1. Except for mastectomy weight, which showed a tendency towards statistical significance $(\mathrm{P}=0.096)$, parameters, such as hypertension, obesity, radiation therapy, endocrine therapy, chemotherapy, and axillary lymph node dissection, showed no statistically significant differences.

Complication rates were compared between the two ADM types to identify any differences. Overall complication rates for thin and thick ADMs were $19.0 \%$ and $23.3 \%$, respectively. Linear regression and binary logistic regression analyses were performed to further understand the impact of ADMs on complication rates. Although complications were slightly more prevalent in the thick ADM group, there was no significant overall difference between groups regarding complications. Details of complications were compared between thin and thick ADM groups (Table 2). The seroma rate was slightly higher for thick ADM group than for thin $\mathrm{ADM}$ group (13.3\% vs. 9.5\%), but there were no statistically significant differences $(\mathrm{P}>0.05)$. Several individual complication groups were analyzed, and no between-group differences regarding rates of flap necrosis, infection, hematoma, cellulitis, and surgical revision were identified.

Despite the lack of difference in complication rates between the two groups, the observed clinical complications during DTI breast reconstruction prompted additional analysis of our data. Univariable and multivariable analyses were performed to identify factors for complications. Factors affecting individual complications and those affecting total complications were analyzed separately. We found statistical significance in patients with higher body mass index (BMI), mastectomy weight, implant volume, 
Table 1 Demographic characteristics

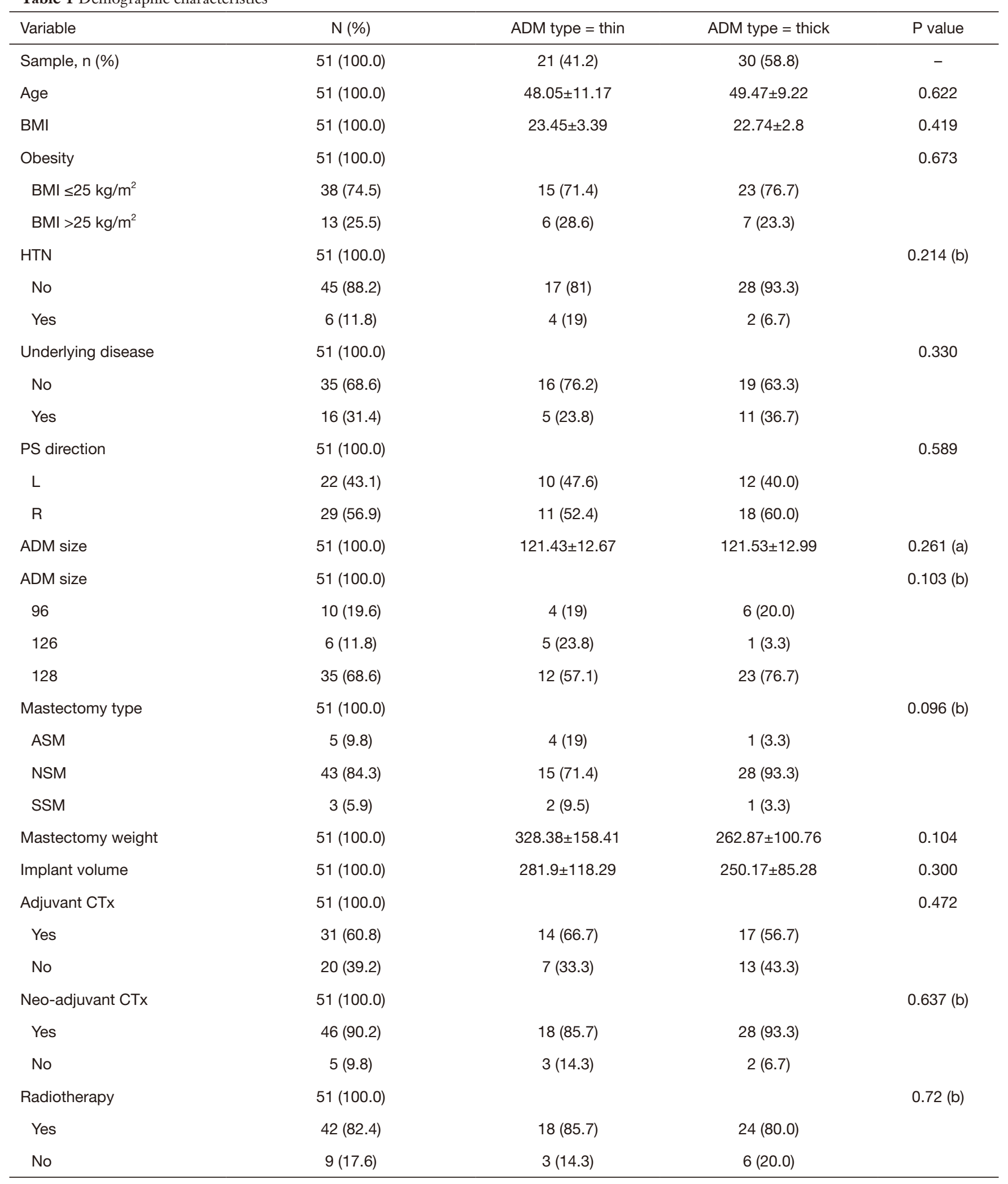

Table 1 (continued) 
Table 1 (continued)

\begin{tabular}{|c|c|c|c|c|}
\hline Variable & N (\%) & ADM type = thin & ADM type = thick & $P$ value \\
\hline Yes & $45(88.2)$ & $19(90.5)$ & $26(86.7)$ & \\
\hline No & $6(11.8)$ & $2(9.5)$ & 4 (13.3) & \\
\hline ALND level & $51(100.0)$ & & & 0.407 (b) \\
\hline 1 & $2(3.9)$ & $0(0)$ & $2(6.7)$ & \\
\hline 2 & $18(35.3)$ & $6(28.6)$ & $12(40.0)$ & \\
\hline
\end{tabular}

Continuous variables were expressed as mean \pm SD. Categoric variables were expressed as sample number and \%. When continuous variables were normally distributed, differences between subgroups were tested using Student's $t$-test or Welch's $t$-test. For non-normally distributed variables, the Mann-Whitney $U$ test was used. For categoric variables, the Chi-squared test or Fisher's exact test was used to compute $\mathrm{P}$ values. $\mathrm{P}$ value (a): computed using Mann-Whitney $\mathrm{U}$ test (i.e., Wilcoxon rank-sum test). $\mathrm{P}$ value (b): computed using Fisher's exact test. ADM, acellular dermal matrix; ALND, axillary lymph node dissection; ASM, areolar-sparing mastectomy; BMI, body mass index; CTx, chemotherapy; HTN, hypertension; NSM, nipple-sparing mastectomy; SSM, skin-sparing mastectomy.

Table 2 Comparison of complications between thin and thick ADMs

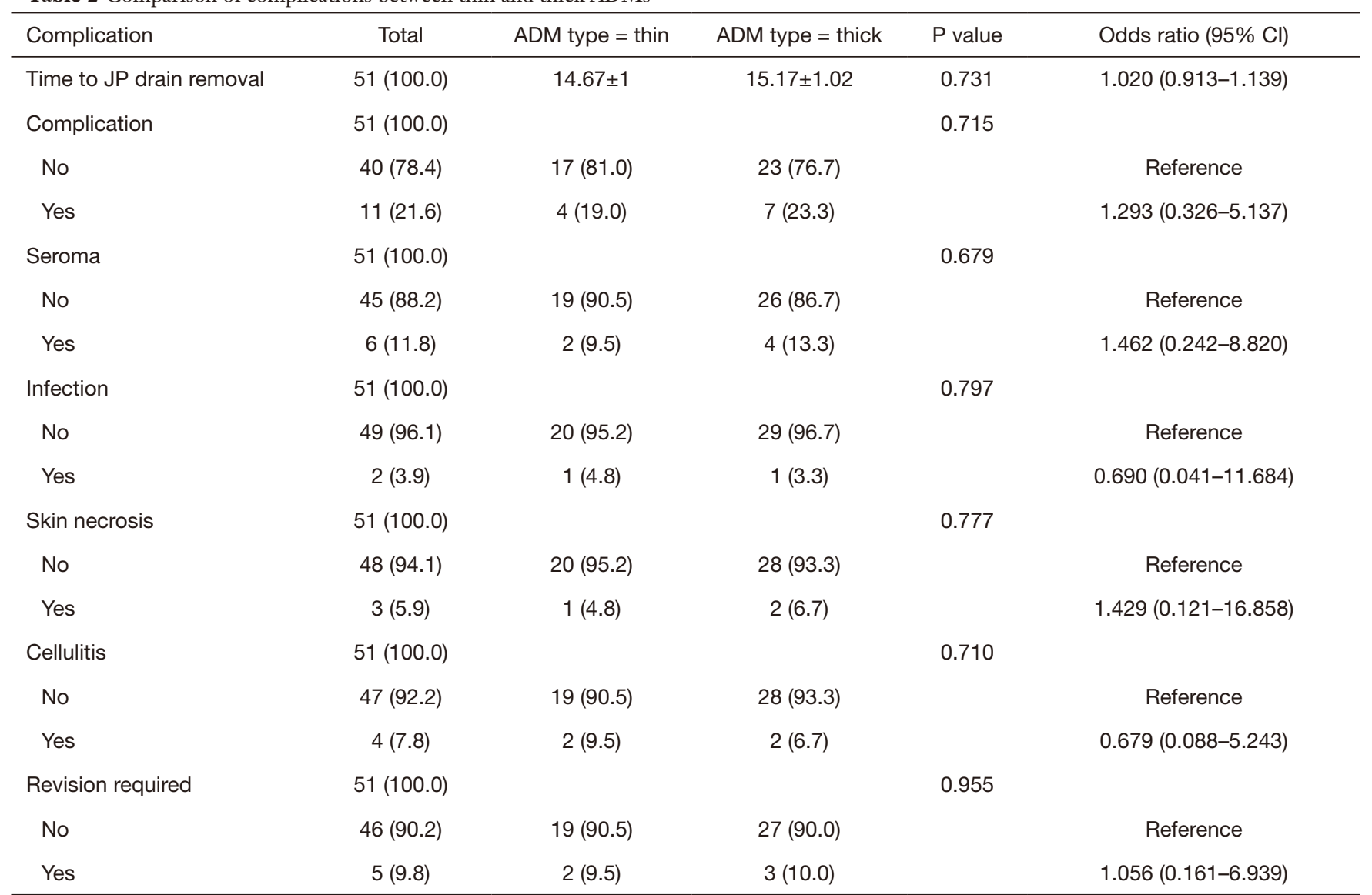

Continuous variables were expressed as mean \pm SD. Categoric variables were expressed as sample number and \%. Odds ratio: computed using binary logistic regression analysis. ADM, acellular dermal matrix; Cl, confidence interval; JP, Jackson-Pratt. 
Table 3 Patient and breast-related characteristics that may have acted as risk factors for time to JP drain removal $(\mathrm{n}=51)$

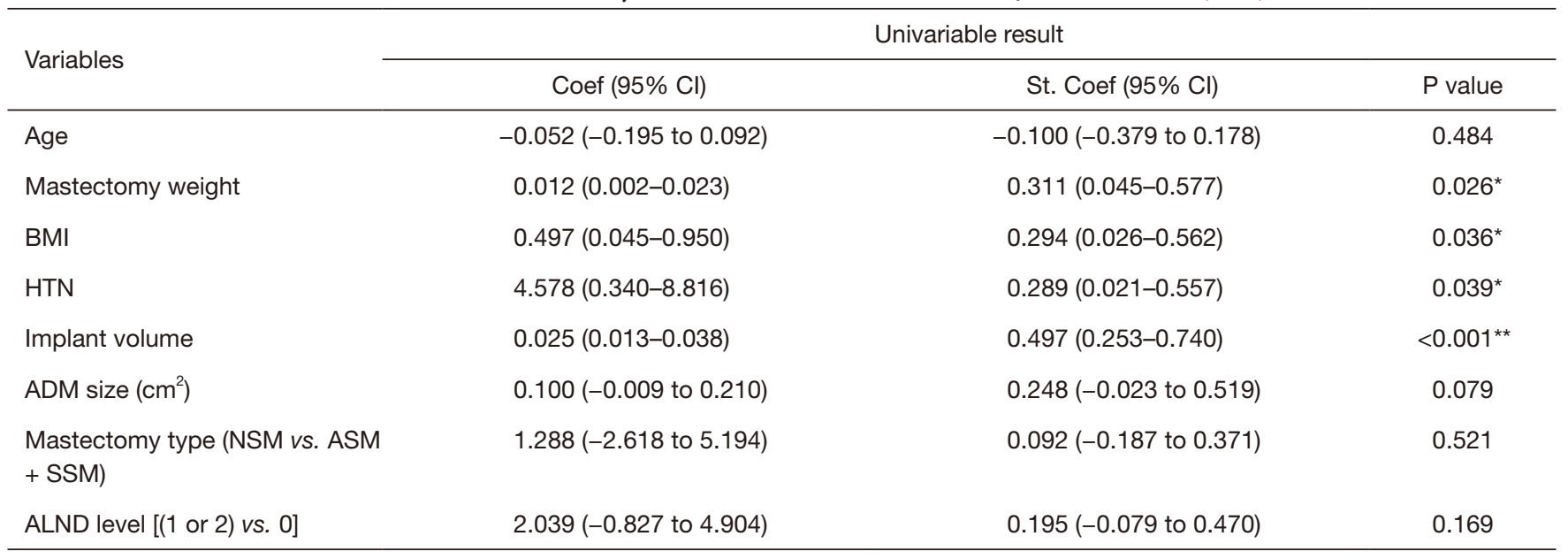

*, $\mathrm{P}<0.05 ;{ }^{* \star}, \mathrm{P}<0.01$. JP, Jackson-Pratt; $\mathrm{Cl}$, confidence interval; Coef, coefficient of linear regression; St. Coef, standardized coefficient of linear regression; BMI, body mass index; HTN, hypertension; ADM, acellular dermal matrix; NSM, nipple-sparing mastectomy; ASM, areolar-sparing mastectomy; SSM, skin-sparing mastectomy; ALND, axillary lymph node dissection.

and hypertension, which prolonged the drain removal time (Table 3). There was also a significantly greater incidence of surgical revision in obese [odds ratio $(\mathrm{OR}) 1.434 ; \mathrm{P}=0.039$ ] and hypertensive patients (OR 21.5; $\mathrm{P}<0.005)$. However, after analysis of the factors affecting other individual complications, significant conclusions were not obtained because the number of complications was too small. On analyzing total complications, there was a greater incidence of complications in obese patients (OR 1.668; $\mathrm{P}=0.047$; Table 4). Regarding patient satisfaction, BREAST-Q yielded similar responses across all metrics between the two groups (Figure 1).

\section{Discussion}

An increase in breast cancer cases inevitably leads to an increase in the need for implant breast reconstruction. However, surgeons are reluctant to perform singlestage breast reconstruction with implants because of the possibility of complications, such as skin necrosis, infections, and implant failure. Thus, some centers have set strict indications, allowing only patients with low BMI and no co-morbidities for immediate single-stage implant breast reconstruction (10). Nevertheless, according to previous reports, DTI reconstruction has several advantages, such as shorter operation time, no additional donor scar, and a simpler surgical technique relative to flap surgery $(11,12)$. Han et al. (13) suggested that single-stage reconstruction is a reliable procedure with lower complications rates than two-stage reconstruction under the same breast skin flap condition. For these reasons, in our center, single-stage breast implant reconstruction was used as first-line option in most cases.

During breast reconstruction, acellular dermal skin substitutes are primarily used in the setting of implant breast reconstruction. The use of $\mathrm{ADM}$ in breast reconstruction improves results by supporting soft tissues and covering the inferior pole of the implant. When ADM is placed, it not only promotes angiogenesis but also functions as a scaffold for new tissue formation, thus resulting in increased mechanical and structural strength (14). The use of ADMs can help recreate natural breast structure and ptosis, thus improving the cosmetic outcome of reconstructed breasts. Previous studies have proven that $\mathrm{ADMs}$ in implant reconstruction can eventually reduce the ischemic skin flap damage by strengthening the covering tissue $(15,16)$ and that they have the potential to minimize adverse inflammation and reduce the incidence of capsular contracture. Komorowska-Timek et al. (17) demonstrated that radiated capsules surrounding implants were strongly associated with inflammatory infiltrates and pseudoepithelial cells, thus leading to prominent capsules, whereas 
Table 4 Patient and breast-related characteristics that may have acted as risk factors for a complicated outcome $(\mathrm{n}=51)$

\begin{tabular}{|c|c|c|c|c|}
\hline Variable (response = complication) & \multicolumn{2}{|c|}{ Univariable result } & \multicolumn{2}{|c|}{ Multivariable result } \\
\hline Age & $1.034(0.966-1.106)$ & 0.334 & $1.055(0.949-1.174)$ & 0.321 \\
\hline Mastectomy weight & $0.999(0.993-1.004)$ & 0.626 & $0.988(0.975-1.002)$ & 0.091 \\
\hline BMI & $1.159(0.927-1.447)$ & 0.195 & $1.668(1.008-2.761)$ & $0.047^{*}$ \\
\hline Yes vs. no & $4.625(0.785-27.246)$ & 0.091 & $1.542(0.108-21.927)$ & 0.749 \\
\hline Implant volume & $1.001(0.995-1.008)$ & 0.683 & $1.004(0.993-1.015)$ & 0.486 \\
\hline ADM size $\left(\mathrm{cm}^{2}\right)$ & $1.151(0.860-1.541)$ & 0.343 & $1.230(0.812-1.863)$ & 0.328 \\
\hline Mastectomy type & & 0.505 & & 0.668 \\
\hline (1 or 2$)$ vs. 0 & $0.507(0.117-2.201)$ & 0.365 & $0.386(0.047-3.144)$ & 0.373 \\
\hline
\end{tabular}

*, $\mathrm{P}<0.05$. OR, odds ratio; $\mathrm{Cl}$, confidence interval; BMI, body mass index; HTN, hypertension; ADM, acellular dermal matrix; NSM, nipplesparing mastectomy; ASM, areolar-sparing mastectomy; SSM, skin-sparing mastectomy; ALND, axillary lymph node dissection.

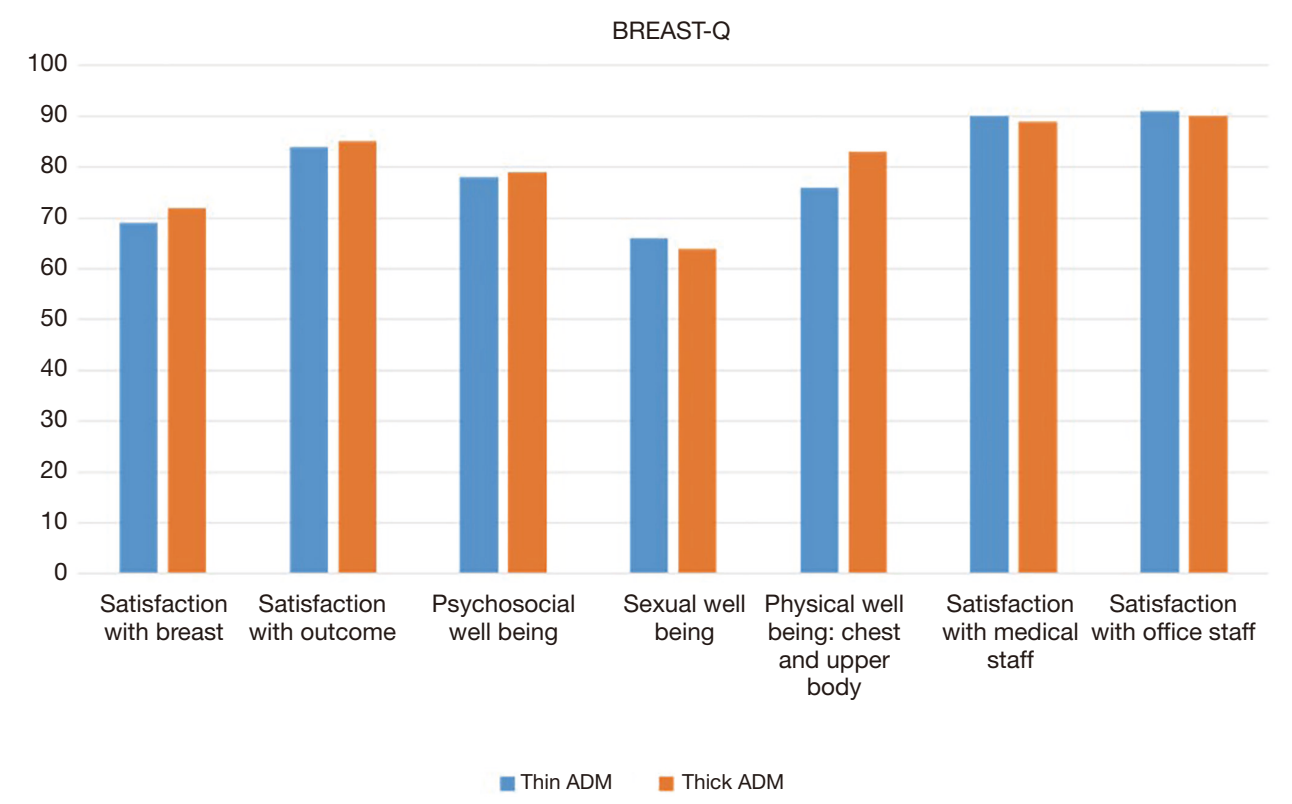

Figure 1 Mean distributions comparing BREAST-Q patient-reported scores from the thin and thick ADM groups. BREAST-Q, Breast Questionnaire; ADM, acellular dermal matrix.

ADM-wrapped implants showed lesser cellular invasion and relatively diminished pseudo-epithelial formation.

Because of these advantages, most plastic surgeons who perform breast reconstruction use ADM. Furthermore, with the advent of the prepectoral breast reconstruction, the importance of using ADM has grown as well. However, various types of ADMs are being produced by different companies, and the characteristics of each product are 


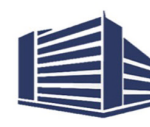

Tissue bank
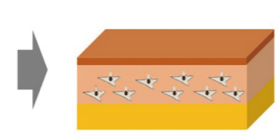

Allograft skin

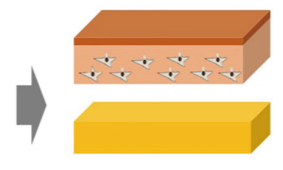

Fat removal

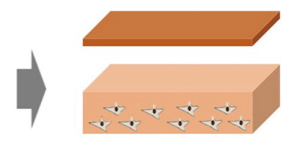

De-epithelization

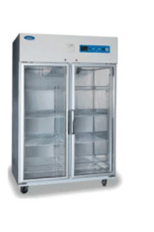

Preservation

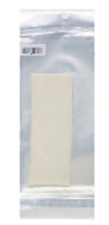

Sterilization

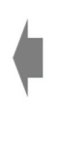

Freeze/drying or hydration

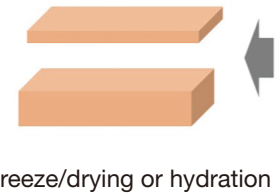

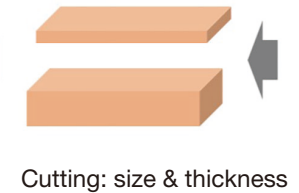

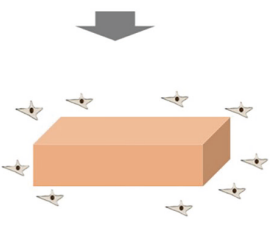

Acellularization

Figure 2 Schematic overview of acellular dermal matrix production.

different. The products vary in many aspects, including the source of tissue, processing, storage, surgical preparation, and available sizes. For example, most ADMs are derived from cadaveric tissues that are decellularized and often terminally sterilized; however, some are also derived from porcine or bovine dermis. However, the choice of which $\mathrm{ADM}$ to use can seem distressingly random. Several studies have reported differences in inherent properties of these products, thus leading to different outcomes (18). Frey et al. (19), in a large retrospective study comparing total sub-muscular reconstruction with three versions of ADM (aseptic, sterile and ready to use, and contoured fenestrated $\mathrm{ADM}$ ) found that the rates of infection for sterile $\mathrm{ADM}$ and fenestrated $\mathrm{ADM}$ were comparable with each other but lower than that of aseptic ADM. Conversely, some studies reported no differences in surgical outcomes despite product differences. Macarios et al. (20) compared sterile and aseptic ADM products in a meta-analysis and found out no statistically significant differences in complication rates. Therefore, the surgeon must first understand the various properties of and differences among the various ADM products, which depend on how immunologically activated cells are removed and packaging methods. Accordingly, the surgeon shall decide which ADM to use.

In addition to these characteristics, ADMs of various thicknesses are being produced. In general, steps involved in preparing an $\mathrm{ADM}$ include harvesting the tissue from a donor (e.g., a human or animal source) and removing the cells under conditions that preserve their biological and structural function. In certain embodiments, the methods of manufacturing Megaderm ${ }^{\circledR}$ (allogeneic cadaveric human dermis) comprise prescreening and testing steps to ensure the safety of the raw materials before cell removal. After basic trimming and cleaning, the allograft skin tissue is placed in a decellularization solution to remove viable cells from the structural matrix without compromising the biological and structural integrity of the collagen matrix and then finally sterilized by e-beam or gamma radiation. After removal of the cell components, the thickness of the $\mathrm{ADM}$ is determined (Figure 2). During these stages, ADM thickness can be adjusted as per customer requirements. Since the price of the product does not change according to the difference in the thickness, the thickness of the $\mathrm{ADM}$ used in the surgery is completely determined by the surgeon's preference. However, studies comparing surgical outcomes with different thicknesses in the same type of $\mathrm{ADM}$ are rare.

The placement of ADM between the skin and the breast implant provides an additional layer covering the implant, and gradually, fibroblast and vessel ingrowth occur, thus integrating ADM into to the recipient breast's subcutaneous tissue. It can augment the breast skin flap thickness and act as a sling to support the implant. Therefore, thicker ADMs are typically expected to support implants more strongly while providing a sense of stability, making the surface smoother, reducing the need for explantation and the incidence of capsular contracture. However, there are ongoing concerns regarding the increased risk of seroma and infection due to $\mathrm{ADM}$, and thicker $\mathrm{ADM}$ s are also expected to be associated with higher seroma rates because rapid bio-integration arrests the acute healing phase and production of serous fluid (21). We can assume that the cellular invasion and vascular ingrowth in thick dermal tissue is a more time-consuming process therefore increase complications. Within the implanted thick ADM, there may be a relatively a longer process, involving the 
ingrowth of blood vessels and recolonization by fibroblasts, myofibroblasts, and various connective tissue free cells (lymphocytes, macrophages, granulocytes, mast cells). In addition, it has been suggested that porous ADMs provide a larger surface area of $\mathrm{ADM} /$ host interface, thus accelerating the integration process, and meshed ADM significantly decreased the time needed for postoperative drain $(22,23)$.

Nonetheless, in this study, ADM thickness did not affect the incidence of complications. Complications occurred slightly more in the thick ADM group, which also required more drain time; however, between-group differences were not statistically significant. Some studies have suggested that the increased risks of serious complications and overall morbidity from using ADM were not significant; moreover, it should be noted that the use of ADM played a role in reducing complications during long-term followup (24). In addition, in studies comparing the results of different types of $\mathrm{ADM}$ (partial-sling vs. full-sling $\mathrm{ADM}$ ) in breast reconstructive surgery using tissue expanders, complication rates did not differ significantly between groups (25). The exact mechanism responsible for no increase in complications despite the insertion of ADM, a foreign substance, remains unknown. However, according to these studies, of course, the incidence of complications may vary depending on the type of ADM, but the using an ADM itself and size of the ADM used do not have a significant relationship with complications. In addition, ADM thickness may also be considered to have no effect on the incidence of complications. Clearly, the present study was a small cohort study with short follow-up duration, and larger, longer-term studies are needed to confirm the complication incidence associated with different thicknesses of ADM. In addition, to further characterize the degree of inflammation, recellularization, and vascularization associated with the thickness of ADM, histologic and immunohistochemical analyses will also be needed.

In this study, obesity and hypertension were significant factors in the development of complications rather than the thickness of ADM. Obesity is a well-known risk factor for complications, causing an increase in dead space and seroma. Some studies have shown that the use of ADM is not recommended for obese patients; therefore, it is necessary to use ADM selectively. However, even in obese individuals, the thickness of ADM was not an additional factor for complications. Actually, their systemic high blood pressure affects the wound healing process and acts as a factor that interferes with normal wound healing. In particular, hypertension has been reported to act as a cause of fibroproliferative disorders caused by promoting fibrogenesis (26). Our study also showed that the close relationship between hypertension and skin fibrosis could be applied to the wound healing process after breast reconstruction using ADM. However, the exact mechanism by which hypertension affects wound healing in breast reconstruction using $\mathrm{ADM}$ and the effects of hypertension controlled by drugs need further study.

Comparing patient satisfaction outcomes between thin and thick ADMs is interesting. Some early reports described subcutaneous breast reconstructions, which fell out of favor due to poor esthetic results related to implant rippling and visibility, poor soft tissue support, increased incidence of capsular contracture, and high incidence of skin flap necrosis (27). Although there are evident improvements in the perfusion of the skin flaps after mastectomy using the minimally disruptive anatomic mastectomy technique and dual plane subpectoral implant placement, the use of ADM also alleviated the problems presenting during subcutaneous breast reconstructions. ADMs were introduced as an efficient and safe option for implant coverage to mask rippling; thus, some surgeons expect superior esthetic outcomes from thicker ADMs. However, our study identified, using BREAST-Q, that there were no statistically significant differences between the two groups regarding esthetic outcomes. The thickness of ADM did not affect the subjective evaluation of the patient. In other words, patient satisfaction outcomes for DTI dual plane subpectoral reconstruction depended mainly on the selection of appropriate implants and the thickness of skin flap and soft tissue remaining after mastectomy and not on the thickness of ADM used. However, this study included only patients with dual plane subpectoral breast implant reconstruction, and further such studies involving cases of prepectoral breast implant reconstruction are needed. In particular, if the implant is placed in the sub-glandular plane, poor tissue coverage over the implant can cause more frequent rippling. If the implant is held up vertically, the shell will naturally cause ripples in the upper pole as the gel falls into the lower part of the implant and the upper part empties. This is because there is an element of fluidity in the gel which makes the implant feel soft. In this case, change from soft silicone to more cohesive filling implant or get more soft tissue cover can solve the problem. It would be possible to change the plane into a submuscular plane underneath the pectoralis major muscle; however, for prepectoral breast reconstruction, thick $\mathrm{ADM}$ may be meaningful.

Our study has potential limitations. Firstly, the 
number of patients was not large when compared with multicenter studies. Secondly, the results were acquired from a retrospective analysis. Thirdly, the follow-up period was insufficient to assess long-term complications, such as capsular contracture. Lastly, because ADMs were cut during surgery to fit the patient's breast size and implant size, the ADM size actually applied to each patient was different. Therefore, the size was not accurately measured during surgery, therefore, the exact size of ADM used during surgery was not reflected in this study. Although well-controlled studies are needed in the future, implantbased breast reconstruction using $\mathrm{ADM}$, regardless of $\mathrm{ADM}$ thickness may be reliable and advantageous.

\section{Conclusions}

As ADM products become more diverse, a lack of research exists about their clinical outcomes. Our study demonstrated that there were no significant differences in terms of complication outcomes and patient satisfaction between thin and thick ADMs; further, there was no impact on the complication rate independent of other patient factors. Of course, the sample size is small, so additional research is needed, but according to our study dual-plane subpectoral implant placement breast reconstruction with inferior $\mathrm{ADM}$ sling regardless of the $\mathrm{ADM}$ thickness is a safe modality that should be considered in any patient who is a candidate for immediate breast reconstruction.

\section{Acknowledgments}

Funding: None.

\section{Footnote}

Reporting Checklist: The authors have completed the STROBE reporting checklist. Available at http://dx. doi. org/10.21037/gs-20-534

Data Sharing Statement: Available at http://dx. doi. org/10. 21037/gs-20-534

Conflicts of Interest: Both authors have completed the ICMJE uniform disclosure form (available at http://dx. doi. org/10. 21037/gs-20-534). Both authors have no conflicts of interest to declare.

Ethical Statement: The authors are accountable for all aspects of the work in ensuring that questions related to the accuracy or integrity of any part of the work are appropriately investigated and resolved. This study was approved by the Institutional Review Board of Ewha Mokdong Hospital (IRB No. 2020-05-043), and was performed in accordance with the principles of the Declaration of Helsinki (as revised in 2013). The requirement of obtaining individual consent was waived for this retrospective analysis.

Open Access Statement: This is an Open Access article distributed in accordance with the Creative Commons Attribution-NonCommercial-NoDerivs 4.0 International License (CC BY-NC-ND 4.0), which permits the noncommercial replication and distribution of the article with the strict proviso that no changes or edits are made and the original work is properly cited (including links to both the formal publication through the relevant DOI and the license). See: https://creativecommons.org/licenses/by-nc-nd/4.0/.

\section{References}

1. Jansen LA, Macadam SA. The use of AlloDerm in postmastectomy alloplastic breast reconstruction: part I. A systematic review. Plast Reconstr Surg 2011;127:2232-44.

2. Korean Breast Cancer Society. 2016 Breast cancer facts \& figures. Seoul: Korean Breast Cancer Society, 2016.

3. Jeevan R, Cromwell DA, Browne JP, et al. Findings of a national comparative audit of mastectomy and breast reconstruction surgery in England. J Plast Reconstr Aesthet Surg 2014;67:1333-44.

4. Juhl AA, Christensen S, Zachariae R, et al. Unilateral breast reconstruction after mastectomy - patient satisfaction, aesthetic outcome and quality of life. Acta Oncol 2017;56:225-31.

5. Harless C, Jacobson SR. Current strategies with 2 -staged prosthetic breast reconstruction. Gland Surg 2015;4:204-11.

6. Vardanian AJ, Clayton JL, Roostaeian J, et al. Comparison of implant- based immediate breast reconstruction with and without acellular dermal matrix. Plast Reconstr Surg 2011;128:403e-410e.

7. Spear SL, Parikh PM, Reisin E, et al. Acellular dermisassisted breast reconstruction. Aesthetic Plast Surg 2008;32:418-25.

8. Seth AK, Persing S, Connor CM, et al. A comparative analysis of cryopreserved versus prehydrated human acellular dermal matrices in tissue expander breast 
reconstruction. Ann Plast Surg 2013;70:632-5.

9. Pusic AL, Klassen AF, Scott AM, et al. Development of a new patient-reported outcome measure for breast surgery: the BREAST-Q. Plast Reconstr Surg 2009;124:345-53.

10. Wink JD, Fischer JP, Nelson JA, et al. Direct-to-implant breast reconstruction: an analysis of 1612 cases from the ACS-NSQIP surgical outcomes database. J Plast Surg Hand Surg 2014;48:375-81.

11. Salzberg CA. Nonexpansive immediate breast reconstruction using human acellular tissue matrix graft (AlloDerm). Ann Plast Surg. 2006;57:1-5.

12. Davila AA, Mioton LM, Chow G, et al. Immediate twostage tissue expander breast reconstruction compared with one-stage permanent implant breast reconstruction: a multi-institutional comparison of short-term complications. J Plast Surg Hand Surg. 2013;47:344-9.

13. Han HH, Kim HB, Kim EK, et al. Direct-to-Implant as a Frontline Option for Immediate Breast Reconstruction: A Comparative Study With 2-Stage Reconstruction. Ann Plast Surg 2018;81:274-9.

14. Ibrahim AM, Koolen PG, Ganor O, et al. Does acellular dermal matrix really improve aesthetic outcome in tissue expander/implant-based breast reconstruction? Aesthetic Plast Surg 2015;39:359-68.

15. Chun YS, Verma K, Rosen H, et al. Implant-based breast reconstruction using acellular dermal matrix and the risk of postoperative complications. Plast Reconstr Surg 2010;125:429-36.

16. Woerdeman LA, Hage JJ, SmeuldersMJ, et al. Skinsparingmastectomy and immediate breast reconstruction by use of implants: an assessment of risk factors for complications and cancer control in 120 patients. Plast Reconstr Surg 2006;118:321-30.

17. Komorowska-Timek E, Oberg KC, Timek TA, et al. The effect of AlloDerm envelopes on periprosthetic capsule formation with and without radiation. Plast Reconstr Surg 2009; 123:807-16.

18. Basu CB, Leong M, Hicks MJ. Acellular cadaveric dermis decreases the inflammatory response in capsule formation

Cite this article as: Hong SE, Kim JH. The relationship of human acellular dermal matrix thickness on complication rate and patient-reported outcomes in implant-based immediate breast reconstruction. Gland Surg 2021;10(1):90-100. doi: $10.21037 /$ gs-20-534 in reconstructive breast surgery. Plast Reconstr Surg 2010;126:1842-7.

19. Frey JD, Alperovich M, Weichman KE, et al. Breast Reconstruction Using Contour Fenestrated AlloDerm: Does Improvement in Design Translate to Improved Outcomes? Plast Reconstr Surg Glob Open 2015;3:e505.

20. Macarios D, Griffin L, Chatterjee A, et al. A meta-analysis assessing postsurgical outcomes between aseptic and sterile AlloDerm regenerative tissue matrix. Plast Reconstr Surg Glob Open 2015;3:e409.

21. Zhao X, Wu X, Dong J, et al. A meta-analysis of postoperative complications of tissue expander/implant breast reconstruction using acellular dermal matrix. Aesthetic Plast Surg 2015;39:892-901.

22. Mangelsdorf S, Vergou T, SterryW, et al. Comparative study of hair follicle morphology in eight mammalian species and humans. Skin Res Technol 2014;20:147-54.

23. Hagarty SE, Yen LL, Luo J, et al. Decreased Length of Postoperative Drain Use, Parenteral Opioids, Length of Stay, and Complication Rates in Patients Receiving Meshed versus Unmeshed Acellular Dermal Matrix in 194 Submuscular Tissue Expander-Based Breast Reconstructions: A Single-Surgeon Cohort Study. Plast Reconstr Surg 2020;145:889-97.

24. Lee KT, Mun GH. Updated Evidence of Acellular Dermal Matrix Use for Implant-Based Breast Reconstruction: A Meta-analysis. Ann Surg Oncol 2016;23:600-10.

25. Lee KT, Eom Y, Mun GH, et al. Efficacy of Partial- Versus Full-Sling Acellular Dermal Matrix Use in Implant-Based Breast Reconstruction: A Head-to-Head Comparison. Aesthetic Plast Surg 2018;42:422-33.

26. Huang C, Ogawa, R. The link between hypertension and pathological scarring: does hypertension cause or promote keloid and hypertrophic scar pathogenesis? Wound Repair Regen 2014;22:462-6.

27. Schlenker JD, Bueno RA, Ricketson G, et al. Loss of silicone implants after subcutaneous mastectomy and reconstruction. Plast Reconstr Surg 1978;62:853-61. 